\title{
Welcome Message from the New Editor-in-Chief
}

It is an honor to serve as editor-in-chief (EiC) for Transactions on the Web (TWEB). I wish to thank the outgoing EiC, Brian Davison, for his service to the journal over the past six years. As always, there are ways to make the journal even better serve the community. Here are some examples of changes I have planned:

- Improve board capacity and diversity, including increasing the size and breadth of the editorial board and creating reviewing opportunities for a more diverse pool of qualified reviewers. The early editorial board additions include Omar Alonso, Craig Macdonald, Debora Donato, Nadia Fawaz, Simon Harper, Eelco Herder (the SIGWEB representative), Polly Huang, Nick Nikiforakis, and Anna Squicciarini. Welcome!

- Broaden awareness, including establishing a social media presence (e.g., you can now follow @acmtransweb on Twitter for journal updates) and finding ways to collaborate with toptier conferences in the field (e.g., conference speaking slots for TWEB authors).

-Increasing the number of submissions, including clarifying the topics of interest on the journal website and soliciting special issue proposals on timely, relevant topics.

- Strengthen connections, including restarting editorial board meetings, sharing regular journal updates with the editorial board for feedback, and strengthening connections with related SIGs.

- Provide more recognition for journal contributors, including initiating outstanding paper awards and awards for outstanding reviewer contributions.

-Finally, I reconfirm TWEB's strong commitment to a fair and timely review process, balancing the needs and constraints of authors, reviewers, and editors.

Thank you to Apu Kapadia and Torsten Suel, who will be stepping down from the TWEB editorial board after five years and six years, respectively. Thank you to the other board members who are continuing their service, many of whom have already devoted a significant amount of time to the journal. To prospective authors, I am looking forward to considering your high-quality submissions on Web-related topics.

Ryen W. White, Ph.D.

Editor-in-Chief, ACM Transactions on the Web

\footnotetext{
ACM Reference format: (May 2021), 1 page.

https://doi.org/10.1145/3456294

(C) 2021 Copyright held by the owner/author(s).

1559-1131/2021/05-ART11e

https://doi.org/10.1145/3456294
}

Ryen W. White. 2021. Welcome Message from the New Editor-in-Chief. ACM Trans. Web 15, 3, Article 11e

ACM Transactions on the Web, Vol. 15, No. 3, Article 11e. Publication date: May 2021. 